\title{
Optimized Pulse Source Employing an Externally Injected Gain-Switched Laser Diode in Conjunction With a Nonlinearly Chirped Grating
}

\author{
P. M. Anandarajah, Member, IEEE, C. Guignard, A. Clarke, Student Member, IEEE, D. Reid, M. Rensing, \\ L. P. Barry, Member, IEEE, G. Edvell, and J. D. Harvey, Member, IEEE
}

\begin{abstract}
In this paper, we demonstrate the generation of transform-limited short optical pulses, which display excellent spectral and temporal qualities by employing a novel technology, based on an externally injected gain-switched laser in conjunction with a nonlinearly chirped grating. Using this technique, 3.5-ps optical pulses exhibiting a time-bandwidth product (TBP) of 0.45 are generated, which are suitable for use in high-speed $80 \mathrm{~Gb} / \mathrm{s}$ optical time-division multiplexing (OTDM) communications systems. The numerical integration of a set of rate equations using suitable parameters for the devices used in the experiments were carried out to further confirm the feasibility of the proposed method for developing an optimized pulse source for high-speed photonic systems.
\end{abstract}

Index Terms-Fiber Bragg gratings (FBGs), optical fiber communication, optical pulse compression, optical pulse generation, semiconductor lasers.

\section{INTRODUCTION}

$\mathbf{T}$ HE increasing demand for media-rich content delivery and the escalation of IP traffic has been fueling the massive growth in demand for bandwidth. This extensive increase in bandwidth usage, which shows no sign of abating in the coming decade, is pushing carriers and service providers to deploy increasing optical backbone transmission capacity. The goal of developing future terabit all-optical communication systems may be achieved by a reduction in channel spacing of wavelengthdivision multiplexed (WDM) systems [1] or an increase in the per-channel data rate. The latter one could be achieved by exploiting electrical time division multiplexing (ETDM) as in WDM/ETDM [2] systems or optical time-division multiplexing (OTDM) [3] as in hybrid WDM/OTDM systems [4].

The ceiling for base data rates in high-speed optical networks for the past few years has been $10 \mathrm{~Gb} / \mathrm{s}$ (OC-192/STM- 64). An attractive alternative to deploying higher wave counts at $10 \mathrm{~Gb} / \mathrm{s}$, taking into account the amount of successful research efforts, is the deployment of higher capacities per wavelength.

Manuscript received October 4, 2005; revised January 27, 2006. This work was supported in part by Science Foundation Ireland (Investigator Program) and Enterprise Ireland (Proof of Concept).

P. M. Anandarajah, C. Guignard, A. Clarke, M. Rensing, and L. P. Barry are with the Research Institute for Networks and Communications Engineering (RINCE), School of Electronic Engineering, Dublin City University, Dublin 9, Ireland (e-mail: anandara@eeng.dcu.ie; guignard@eeng.dcu.ie; clarkea@eeng.dcu.ie; marc.rensing@eeng.dcu.ie; barry1@eeng.dcu.ie).

D. Reid and J. D. Harvey are with the Physics Department, University of Auckland, Auckland 1020, New Zealand (e-mail: d.reid@southernphotonics. com; j.harvey@auckland.ac.nz).

G. Edvell is with Redfern Optical Components, Eveleigh NSW 1430, Australia (e-mail: G.Edvell@redferncomponents.com).

Digital Object Identifier 10.1109/JSTQE.2006.872057
For example, the $40 \mathrm{~Gb} / \mathrm{s}(\mathrm{OC}-768 / \mathrm{STM}-256)$ solution provides better spectral efficiency and results in lower overall cost for capacity, relative to existing $10-\mathrm{Gb} / \mathrm{s}$ systems. The higher line rate also provides better terminal density, which results in smaller terminals for a given capacity [5], [6]. One of the remarkable factors, with the move to higher line rates, is the coding used at the transmitter. Most of the current systems, $2.5-10 \mathrm{~Gb} / \mathrm{s}$, have tended to employ Non-return-to-zero (NRZ) coding. However, to achieve line rates of $40 \mathrm{~Gb} / \mathrm{s}$ and higher, it may become necessary to use return-to-zero (RZ) coding. RZ (pulse) modulation formats offer a number of advantages over NRZ modulation schemes, especially in long-haul transmission, which result in higher signal-to-noise ratio and lower system bit error rate translating into better overall system performance [7]-[10].

The rigorous requirements placed on the transmitter performance by the reduced channel spacing and increased line rate act as an immense challenge to manufacturers. Hence, the design of an optical transmitter, capable of generating pulses with adequate temporal and spectral purity for acceptable operation in high-speed optical communication systems, is crucial. There are numerous methods available to generate picosecond optical pulses [4]. Some of the most common and commercially available optical pulse sources are mode-locked semiconductor and fibre ring lasers [11], [12]. However, it has widely been accepted that the gain-switching technique is one of the simplest and most reliable in comparison to the rest [13], [14]. While the advantages in employing this method are numerous, one of its major drawbacks is the spectral purity of the generated pulses. The direct modulation of the laser diode causes a time-varying carrier density in the active region of the device, which in turn causes a variation in the output wavelength from the laser during the emission of the optical pulse. This results in a frequency chirp across the pulse, which degrades the performance of these pulses when used in practical optical communication systems [15]. It has been reported how this chirp can be used to compress the pulses using dispersion-compensating fibre [16] or linearly chirped gratings [17], to obtain near transform-limited pulses. However, because of the chirp being nonlinear across the pulse, this compression typically results in pedestals on either side of the pulses that make them unsuitable for use in practical systems. By using more complex arrangements involving nonlinear loop mirrors or external modulators, after the linearly compressed pulse, it is possible to greatly reduce the pedestal [18]. Degradation of the side-mode suppression ratio (SMSR) and a relatively large temporal jitter are other inherent problems associated with 
the technique of gain switching. However, by externally injecting into a gain-switched laser, the above-mentioned shortcomings could be overcome [18], [19].

The work described in this paper concerns the generation of temporally and spectrally pure short optical pulses (temporal pedestal, and spectral SMRSs greater than $30 \mathrm{~dB}$ ). The pulse source comprises a nonlinearly chirped fiber Bragg grating (NC FBG) in conjunction with an externally injected gain-switched laser [20]. The design of the NC FBG is determined by the complete characterization of the gain-switched pulse using the technique of frequency resolved optical gating (FROG) [21]. This characterization yields the parameters that are required for the design of an NC FBG with a group-delay profile that is opposite to that measured across the pulse. By employing the tailor-made NC FBG after the gain-switched laser, we can achieve direct compression of the gain-switched pulses to obtain near transform-limited pulses. We show experimental results, obtained by employing this novel technique, where 3.5-ps pulses (FWHM) at a repetition rate of $10 \mathrm{GHz}$ and an associated spectral width of $130 \mathrm{GHz}$ are generated. The resulting pulse and spectral widths show that this technique yields near transform-limited pulses with a time-bandwidth product (TBP) of 0.45 . These pulses also portray an extinction of the temporal pedestals up to about $35 \mathrm{~dB}$ below the peak of the pulse. This excellent temporal pedestal suppression ratio (TPSR) is achieved not only by the grating filter having a nonlinear group-delay profile that is the inverse of that across the gain-switched pulse directly from the laser, but also by ensuring that the grating filter has a specially adapted filter-transfer characteristic. The NC FBG has a custom-designed nonlinear reflective profile that optimizes the output pulse spectrum thereby further enhancing the temporal quality of the pulses. The spectral and temporal characteristics of such a pulse source would make it suitable for use as a transmitter in $80 \mathrm{~Gb} / \mathrm{s}$ OTDM or hybrid WDM/OTDM systems.

This paper is divided into four main sections as follows. Section II focuses on the layout of the externally injected gainswitched laser and the complete characterization of the generated pulses. Section III then concentrates on the fabrication of two types of gratings that could be used to compress the externally injected gain-switched pulses. The employment of the two types of gratings to achieve pulse compression is explained in Section IV. Finally, Section V presents the numerical modeling carried out to validate our experimental results.

\section{EXTERNALLY INJECTED GAIN-SWITCHED LASER}

The experimental setup employed in this work is shown in Fig. 1. A 10-GHz sine wave is amplified with the aid of a highpower RF amplifier. A bias tee is then used to combine the electrical RF signal with a dc bias $\left(2.5 I_{\mathrm{th}}\right)$ to enable gain switching of a commercially available NEL DFB laser contained within a hermetically sealed high-speed package. The laser used has a $3-\mathrm{dB}$ bandwidth of $20 \mathrm{GHz}$, and an output power of $4.7 \mathrm{dBm}$, both measured at a bias current of $3 I_{\mathrm{th}}$. The resulting pulses generated were at a wavelength of $1549.35 \mathrm{~nm}$. Wavelength tun-

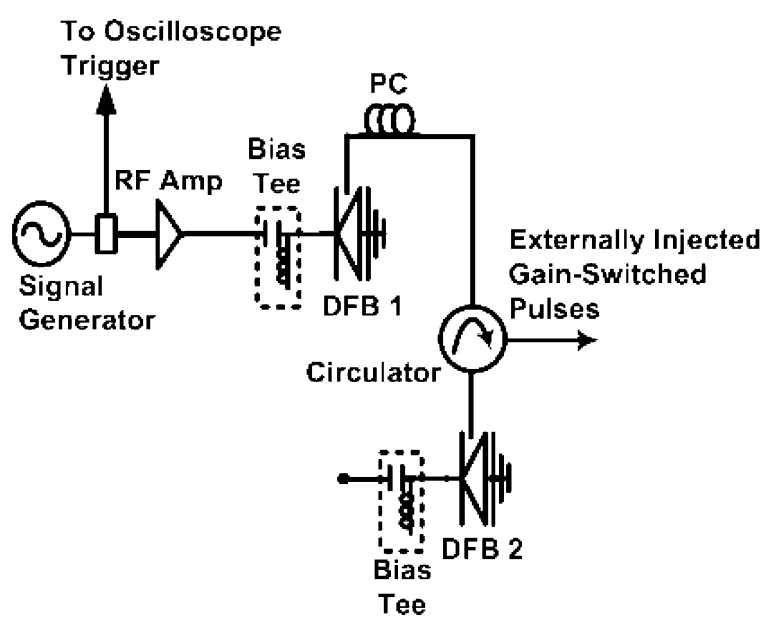

Fig. 1. Experimental setup for pulse generation using an externally injected gain-switched laser.

ability of the laser mode over a range of $2 \mathrm{~nm}$ could be achieved by temperature controlling the diode.

To overcome the poor SMSR $(\sim 5 \mathrm{~dB})$ and timing jitter $(\sim 2$ ps) of the gain-switched pulses, we use external injection (via an optical circulator) from a second DFB (2) laser biased at $23.5 \mathrm{~mA}\left(\sim 1.2 I_{\mathrm{th}}\right)$. A polarization controller was also used to ensure that the light being injected was aligned with the optical axis of the modulated laser. The injected power, incident on the modulated laser diode, was measured to be about $-20 \mathrm{dBm}$ after considering the losses incurred in the optical injection path. External light injection improves the SMSR to around $30 \mathrm{~dB}$ and reduces the timing jitter to $<1$ ps (as measured using an Agilent Digital Communications Analyzer). The actual jitter on the externally injected gain-switched output is expected to be in the order of 200 fs or less as demonstrated in previous work [22]. The generated pulses can then be characterized using an optical spectrum analyzer (OSA), a high-speed oscilloscope in conjunction with a $50-\mathrm{GHz}$ pin detector, and also a FROG measurement system.

Fig. 2(a) and (b) displays the optical spectra of the gainswitched laser both without and with external injection, respectively, and it can be seen that the case without injection results in a degraded SMSR of about $5 \mathrm{~dB}$. The overlapping of the broadened modes prevents the side mode being distinguished from the main mode. However, with external injection, a vast improvement in the SMSR ( $>30 \mathrm{~dB})$ is evident. Moreover, from the same figure, the spectral width of the externally injected gain-switched laser is determined to be about $140 \mathrm{GHz}$. Fig. 3 shows the nonaveraged oscilloscope trace of the detected pulse from which the low temporal jitter can be noted. The ringing in this pulse is due to its duration being shorter than the response time of the detector. To accurately characterize this pulse, it is thus necessary to use the FROG technique.

In these experiments, a standard second harmonic generation (SHG) FROG based on the spectral resolution of the output from a noncollinear autocorrelator, as described in [21], was used. SHG was performed in a beta barium borate (BBO) crystal with an interaction length of $250 \mu \mathrm{m}$. With this interaction length, the expected variation in the SHG response [23] was 


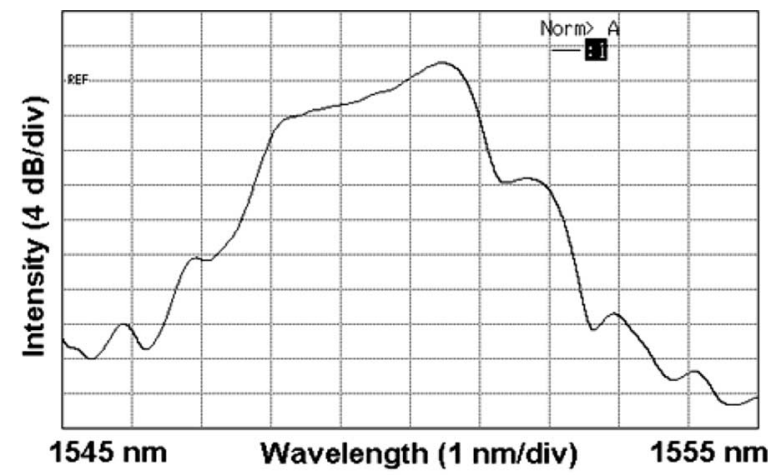

(a)

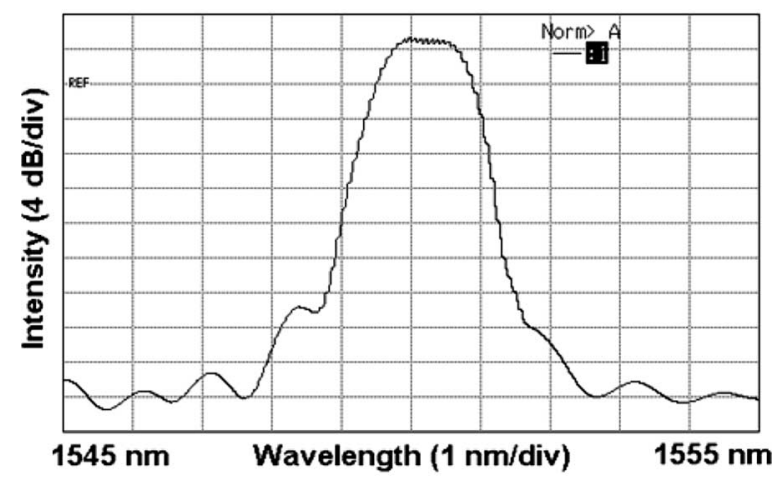

(b)

Fig. 2. Spectra of gain-switched laser (a) without and (b) with injection.

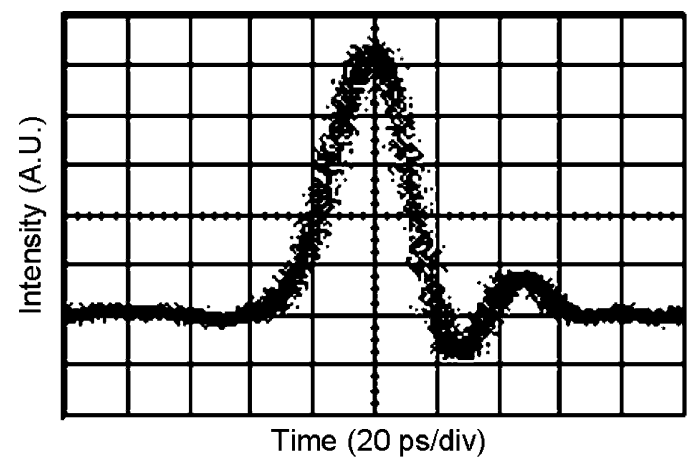

Fig. 3. Oscilloscope trace of the externally injected gain-switched pulse.

negligible over a 100-nm bandwidth around $1550 \mathrm{~nm}$. The SHG output was spectrally resolved using a grating spectrometer with a 1024-element cooled photodiode array mounted on the output. The spectral resolution was $\Delta \lambda=0.04 \mathrm{~nm}$ at an SHG wavelength of $775 \mathrm{~nm}$. The autocorrelator delay was controlled by a stepping motor with temporal resolution of $\Delta \tau=6.7 \mathrm{fs}$. The complete electric field of the input optical pulse can be determined from the measured spectrogram by using standard retrieval techniques [21]. A short-pulse erbium-doped fibre amplifier (EDFA), specifically designed for the amplification of pulses with a duration in the order of 2 ps (FWHM), is used before the FROG measurement setup to improve the signal-tonoise ratio of the measurement. From the FROG measurement, we can accurately characterize the intensity and chirp profile across the optical pulses from the gain-switched laser with external injection. Fig. 4 indicates that the pulses had a duration

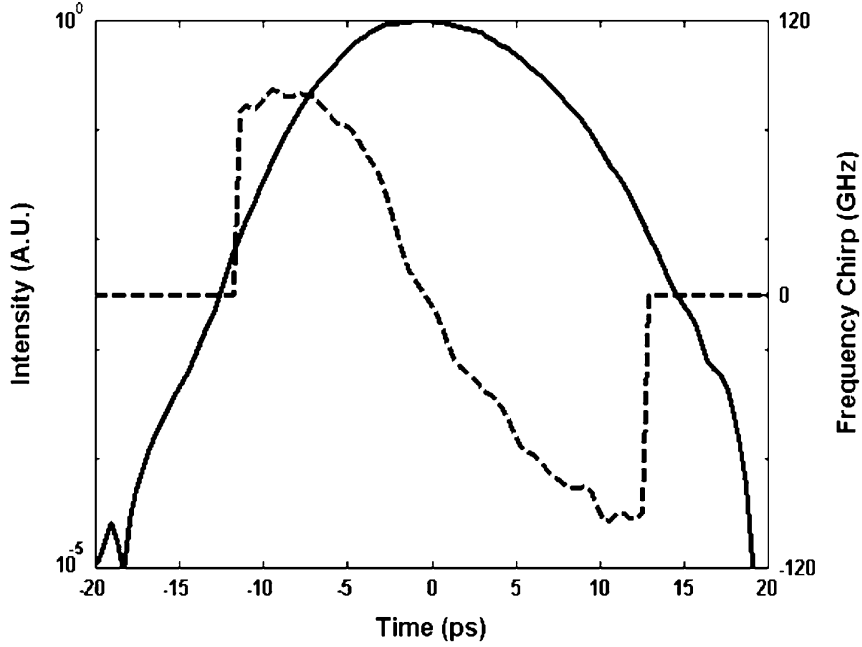

Fig. 4. Intensity (solid line) and chirp (dashed line) of optical pulses from the externally injected gain-switched laser.

(FWHM) of about $10.5 \mathrm{ps}$ and that the frequency chirp (dashed line) becomes nonlinear in the wings of the pulse generated due to the gain-switching mechanism. The resulting TBP of the pulse is 1.5 .

\section{GRATING FABRication}

We subsequently use the measured nonlinear chirp across the pulse to design and fabricate an NC FBG. This process involves the initial creation of the group-delay response for the FBG based on the group-delay data derived from the FROG measurements of the externally injected gain-switched pulse. The FBG target group-delay response is simply selected as the inverse of the pulse group-delay response, which should result in the pulse having a constant group-delay profile over the pulse bandwidth after it has reflected from the FBG. In addition to a constant group-delay profile across the pulse bandwidth, for an optimized pulse source, we also require the pulse to exhibit a Gaussian spectrum. Generally, gain-switched spectra tend to be more rectangular than Gaussian spectra. The reflection profile of the NC FBG is constructed as the difference between the spectral amplitude of the gain-switched output and a Gaussian profile, which should result in the compressed pulse portraying a Gaussian spectrum. Once the FBG target spectrum and group-delay profile are obtained, it is relatively straightforward to calculate an FBG design that can be implemented into the optical fibre by using an inverse scattering algorithm [24]-[26]. The FBGs are fabricated by using a holographic writing method [27], where a computer electronically controls the FBG phase and amplitude and does not require a custom-made phase mask. Hence, it is even possible to fabricate NC FBGs for each individual DFB laser in larger volumes at a low cost (as there is no need for a custom-made phase mask), if the nonlinear chirp is different from laser to laser.

The reflective and group-delay profiles of the fabricated NC FBG are shown in Fig. 5. We also fabricated a linearly chirped fibre grating that had a chirp profile opposite to a linear approximation of the chirp across the gain-switched pulse. In both 


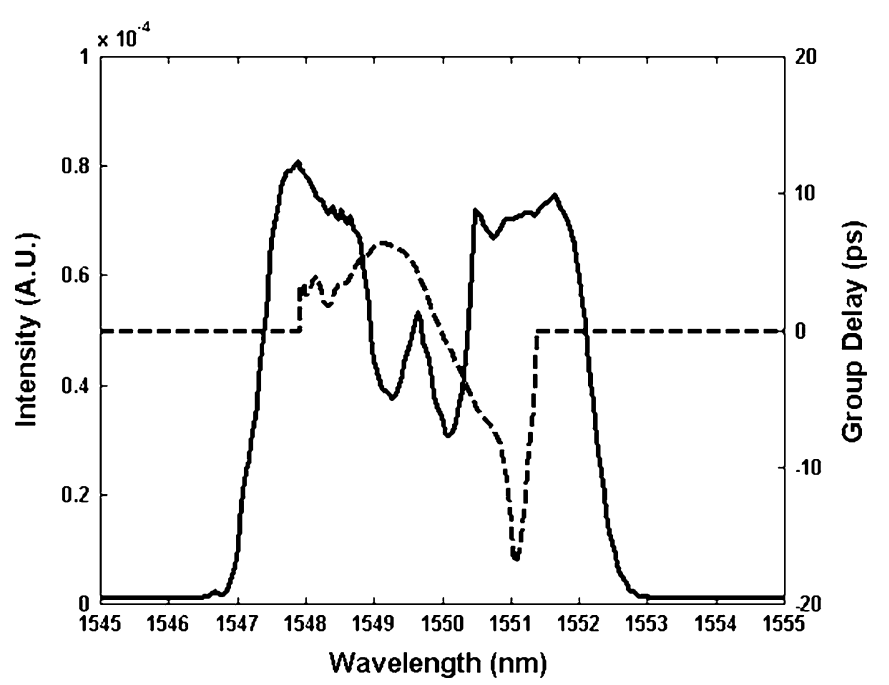

Fig. 5. Reflection (solid line) and group delay (dashed line) profiles of the nonlinearly chirped fiber grating.

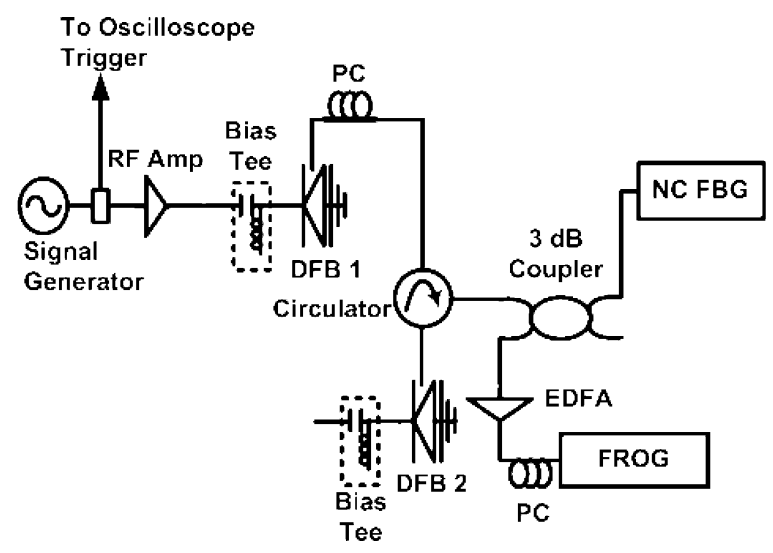

Fig. 6. Experimental setup for optimized pulse generation.

the cases, the agreement between the measured and the target group-delay profile was very good, with a standard deviation of the measured error $<1.5 \mathrm{ps}$, which is quite close to the estimated noise level of $+/-1$ ps for the group-delay measurements.

\section{Pulse COMPREssion}

By placing the nonlinear and linear fibre gratings after the externally injected gain-switched laser, as shown schematically in Fig. 6, we subsequently characterize the pulse compression in the fibre gratings using the FROG technique. In the experimental work presented here, the pulse recovery routinely gave retrieval errors of $G<0.005$ on a $128 \times 128$ grid [21], which indicated the accuracy of our retrievals.

Fig. 7(a) and (b) shows the measured intensity and chirp profile of the gain-switched optical pulses after compression with the linearly and nonlinearly chirped fibre gratings, respectively. In both cases, the gratings have eliminated any frequency chirp across the center of the pulses. However, when the linearly chirped grating is used, we can see how the nonlinearity of the chirp directly from the gain-switched laser results in significant pedestals on the leading and trailing edges of the pulse

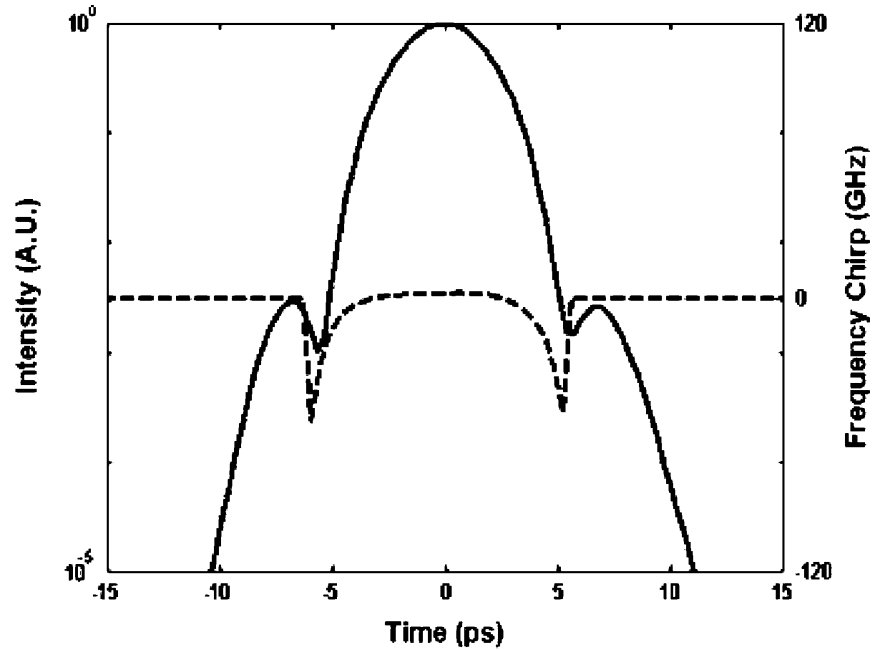

(a)

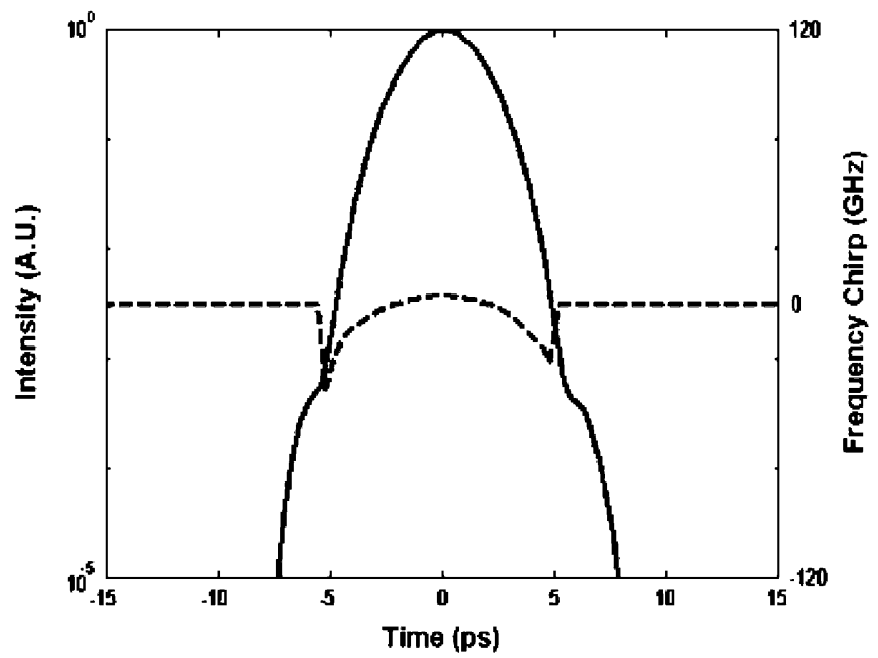

(b)

Fig. 7. Intensity (solid line) and chirp (dashed line) profiles of externally injected gain-switched pulses after (a) linearly chirped and (b) nonlinearly chirped gratings.

[Fig. 7(a)]. Such pedestals, which are around $23 \mathrm{~dB}$ down from the peak of the pulse, would clearly pose significant problems (through intersymbol interference) for the use of these pulses in high-speed OTDM systems [28].

The compression in the nonlinear fibre grating results in a 3.5-ps FWHM pulse [pulse and corresponding chirp profile shown in Fig. 7(b)]. It can clearly be seen that the resultant chirp is flat and has a very small order of magnitude across the pulse. Also, as can be seen in the figure, the pedestals have almost been eliminated (TPSR $>35 \mathrm{~dB}$ ). The latter one is due to not only the elimination of the chirp across the pulse but also the output pulse spectrum being Gaussian due to the nonlinear reflection profile of the grating, as explained earlier. It is important to note that, for a non-Gaussian spectrum, even if the group delay is entirely compensated, temporal pedestals could exist. The Fourier transform of a rectangular spectrum, which results in a Sinc temporal pulse [29], could be used to justify the presence of such pedestals. It is also important to note that a large group delay ripple (GDR) in the compressing FBG could cause 


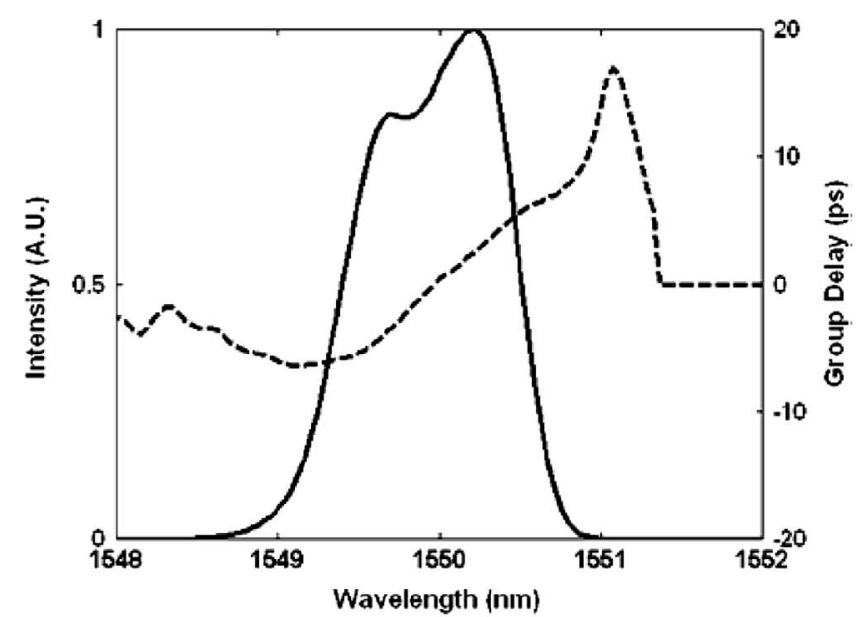

(a)

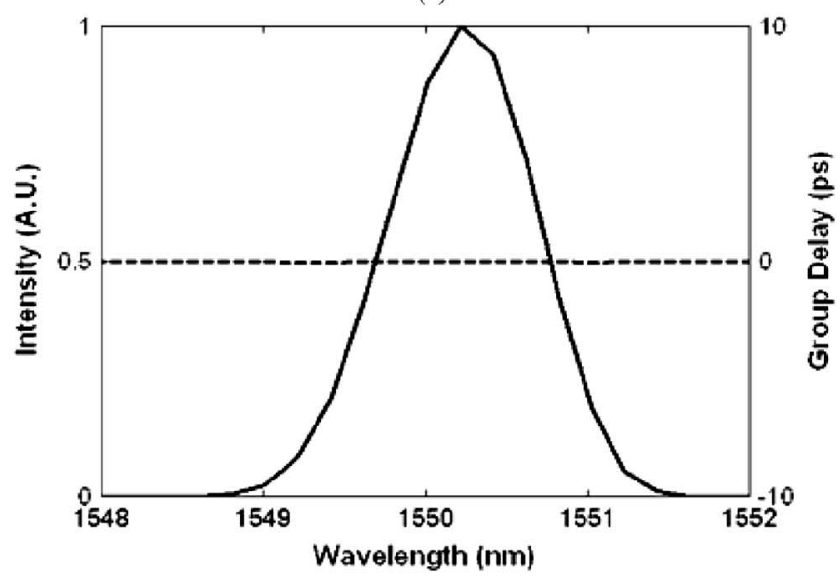

(b)

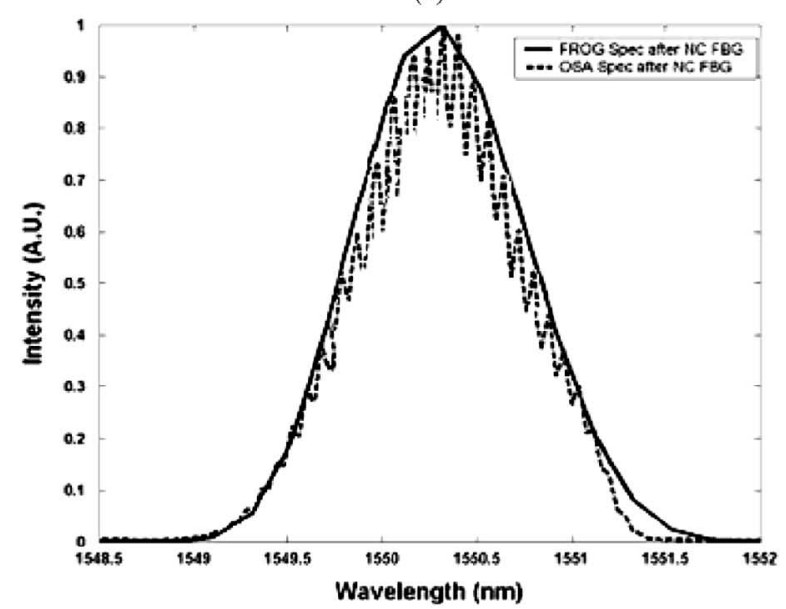

(c)

Fig. 8. (a) The input to and (b) output spectrum (solid line) from the NC FBG and their corresponding group delays (dashed lines) and (c) a comparison of the spectrum obtained by the FROG (solid line) and the OSA (dashed line) after the NCFBG.

low-intensity temporal pedestals. However, in this experiment, no such pedestals were observed mainly due to the low level of GDR, as mentioned earlier, with a standard deviation of less then half the compressed pulsewidth.

The spectra and the group delay of the input and output pulses to and from the NC FBG are shown in Fig. 8(a) and (b), respec- tively. It is clear that the group delay has been compensated for entirely by the tailor-made NC FBG. The output spectrum is more Gaussian shaped and symmetric in comparison to the input, which is due to the compensation by the nonlinear reflection profile of the NC FBG. The OSA spectrum, which is in excellent agreement with the pulse spectrum obtained from the FROG measurement, shows that the spectral width is around $130 \mathrm{GHz}$ as shown in Fig. 8(c). The quoted pulsewidth and associated spectral width result in a TBP of 0.45 .

This pulse generation/compression technique portrays excellent repeatability. Furthermore, within laboratory conditions, the scheme exhibited stable operation over about a 24 hour period. This could be mainly attributed to the bias current and temperature of the two DFB (modulated and seeding) lasers being controlled with the aid of Profile current/temperature controllers. Hence, drifts in wavelength of the lasers, due to current or temperature variations were negligible. Furthermore, the wavelength variation with temperature of the fabricated FBGs being relatively small $\left(\sim 0.009 \mathrm{~nm} /{ }^{\circ} \mathrm{C}\right)$ also leads to the stable generation of optimized pulses over very long periods of time.

\section{Simulations}

\section{A. Theoretical Model}

To further confirm the effect of both the group-delay and the reflectivity profiles of the FBG on the pulse compression and to validate the experimental results, numerical simulations were carried out. The laser is described by the following single-mode rate equations [30], [31], which are given for the carrier density $N(t)$ and the complex electric field $E(t)=\sqrt{S(t)} e^{i(\omega t+\varphi(t))}$, where $S(t)$ is the photon density and $\varphi(t)$ is the phase

$$
\begin{aligned}
\dot{E}(t)= & \frac{1}{2}\left[\Gamma_{c} g(N, S)-\frac{1}{\tau_{\mathrm{p}}}\right] E(t) \\
& +i \frac{\alpha_{\mathrm{H}}}{2}\left[\Gamma_{\mathrm{C}} G_{\mathrm{N}}\left(N(t)-N_{\mathrm{t}}\right)-\frac{1}{\tau_{\mathrm{p}}}\right] E(t) \\
\dot{N}(t)= & J-\frac{N(t)}{\tau_{\mathrm{e}}}-g(N, S) S(t) .
\end{aligned}
$$

These equations call for the physical constants described below. $N_{\mathrm{t}}$ and $N_{\mathrm{th}}$ are, respectively, the carrier density at transparency and at threshold with $N_{\mathrm{th}}=N_{\mathrm{t}}+\left(1 / \Gamma_{\mathrm{C}} G_{\mathrm{N}} \tau_{\mathrm{p}}\right) . \Gamma_{\mathrm{C}}$ is the field confinement factor, $G_{\mathrm{N}}$ is the differential gain, and $\alpha_{\mathrm{H}}$ is the linewidth enhancement factor. $\omega_{0}$ is the angular frequency of the solitary laser at threshold. The nonlinear gain is expressed as $g(N, S)=G_{\mathrm{N}}\left(N(t)-N_{\mathrm{t}}\right)\left(1-\varepsilon_{\mathrm{nl}} S(t)\right)$, where $\varepsilon_{\mathrm{nl}}$ is the gain compression factor. The carrier lifetime $\tau_{\mathrm{e}}$ is determined at threshold by $1 / \tau_{\mathrm{e}}=A+B N_{\mathrm{th}}+C N_{\mathrm{th}}^{2}$ with $A$ the rate of nonradiative recombinations, $B$ the rate of spontaneous radiative recombinations, and $C$ the rate of Auger recombination processes. The photon lifetime $\tau_{\mathrm{p}}$ is determined by the full losses of the solitary laser: $1 / \tau_{\mathrm{p}}=v_{\mathrm{g}} \alpha_{\mathrm{in}}-1 / \tau_{\mathrm{c}} \ln \left(R_{1} R_{2}\right)$. Here, $\alpha_{\text {in }}$ corresponds to the scattering losses in the active volume, $v_{\mathrm{g}}=c / n_{\mathrm{g}}$ is the group velocity with $c$ the speed of light in vacuum and $n_{\mathrm{g}}$ the group index of the active medium. The parameters $R_{1}$ and $R_{2}$ are the power reflectivity of the left and 
TABLE I

SOME PARAMETER VALUES FOR THE LASER DiOdE USED IN THE NUMERICAL SimUlationS

\begin{tabular}{|l|l|}
\hline Physical constant & Value \\
\hline Length of the active medium $\left(L_{\mathrm{D}}\right)$ & $3 \times 10^{-4} \mathrm{~m}$ \\
\hline Width of the active medium $(l)$ & $9 \times 10^{-7} \mathrm{~m}$ \\
\hline Thickness of the active medium $(e)$ & $8.5 \times 10^{-8} \mathrm{~m}$ \\
\hline Group index of the active medium $\left(n_{\mathrm{g}}\right)$ & 3.57 \\
\hline Scattering losses $\left(\alpha_{\text {in }}\right)$ & $4020 \mathrm{~m}^{-1}$ \\
\hline Confinement factor $\left(\Gamma_{\mathrm{C}}\right)$ & 0.51 \\
\hline Differential gain $\left(G_{\mathrm{N}}\right)$ & $5.2 \times 10^{-20} \mathrm{~m}^{-2}$ \\
\hline Gain compression factor $\left(\varepsilon_{\mathrm{nl}}\right)$ & $5.2 \times 10^{-20} \mathrm{sm}^{3}$ \\
\hline Carrier density at transparency $\left(N_{\mathrm{t}}\right)$ & $1.0 \times 10^{-24} \mathrm{~m}^{-3}$ \\
\hline Rate of non-radiative recombinations $(A)$ & $2.7 \times 10^{6} \mathrm{~s}^{-1}$ \\
\hline Rate of spontaneous radiative recombinations $(B)$ & $9 \times 10^{-17} \mathrm{~s}^{-1} \mathrm{~m}^{-3}$ \\
\hline Rate of Auger recombination processes $(C)$ & $1.8 \times 10^{-39} \mathrm{~s}^{-1} \mathrm{~m}^{-6}$ \\
\hline Carrier lifetime at threshold $\left(\tau_{\mathrm{e}}\right)$ & $0.31 \mathrm{~ns}^{-1}$ \\
\hline Round trip time in laser cavity $\left(\tau_{\mathrm{c}}\right)$ & $7.14 \mathrm{ps}^{-}$ \\
\hline Linewidth enhancement factor $\left(\alpha_{\mathrm{H}}\right)$ & 2 \\
\hline Amplitude reflectivity $\left(R_{1}\right)$ & 0.3 \\
\hline Amplitude reflectivity $\left(R_{2}\right)$ & 0.3 \\
\hline
\end{tabular}

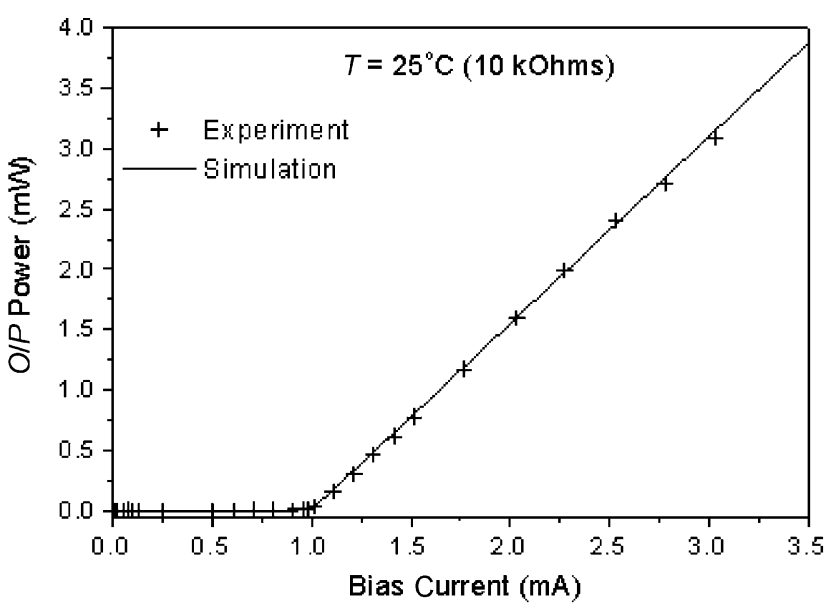

(a)

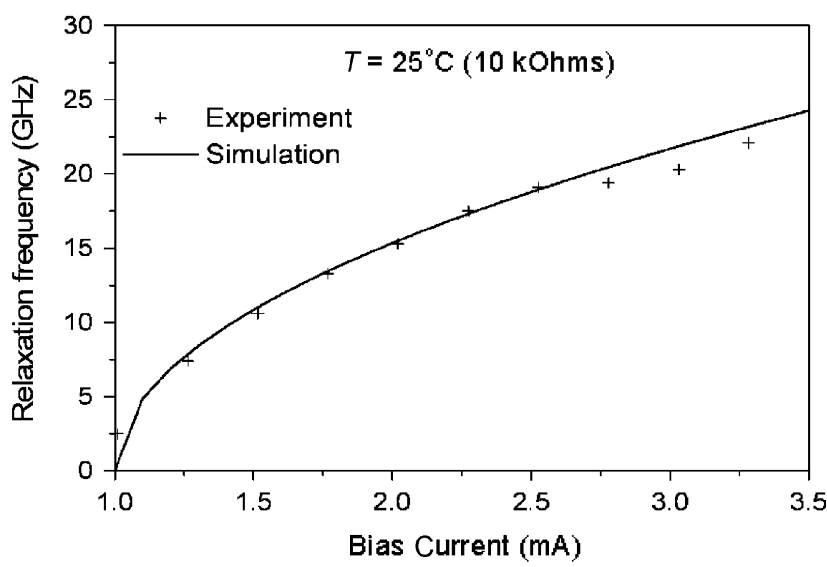

(b)

Fig. 9. (a) Experimental and numerical curves for PI and (b) the evolution of the relaxation frequency against the bias current. right facets of the laser: $R_{1,2}=r_{1,2}^{2} \cdot \tau_{c}=2 L_{\mathrm{D}} / v_{\mathrm{g}}$ corresponds to the cavity lifetime and $L_{\mathrm{D}}$ is the laser diode length. Finally, $J=(I / q V)$ is the injection current density with $V$ the volume of the active medium. When the laser is gain switched, its current consists of the bias $I_{\mathrm{b}}$ and the modulation current $\Delta I(t) . I(t)=I_{\mathrm{b}}+\Delta I(t)=I_{\mathrm{b}}+m \sin \left(2 \pi f_{\mathrm{m}} t\right)$, where $m$ corresponds to the modulation depth and $f_{\mathrm{m}}$ to the modulation frequency.

Table I gives the values of the parameters we used in our simulations. Most of them were provided by the constructor, while a few such as the nonradiative recombination and the Auger recombination process were estimated from experimental results. Fig. 9 displays both the experimental and theoretical graphs for the $P I$ curve and the evolution of the relaxation frequency against the bias current. The good agreement between the experimental and theoretical results (both static and dynamic characteristics) demonstrates that our model is reasonably accurate.

To simplify the study, we have not considered external optical injection in these simulations. In fact, the low level of injection, used experimentally, should not have great influence on the chirp. The sole reason for injection is to improve the SMSR and jitter. As a consequence, the simulation results presented can be easily compared and contrasted to the experimental results.

\section{B. Results}

Setting the bias current $I_{\mathrm{b}}$ at $2 I_{\mathrm{th}}$ and the modulation frequency at $10 \mathrm{GHz}$, we examined the effect of filtering the gain-switched pulse with both linearly and nonlinearly chirped FBG, when the reflection profile of the grating was either square topped, or had a nonlinear profile to achieve a Gaussian spectral output. In the simulations, (1) and (2) were solved directly using a fourth-order Runge-Kutta algorithm. The initial conditions are 


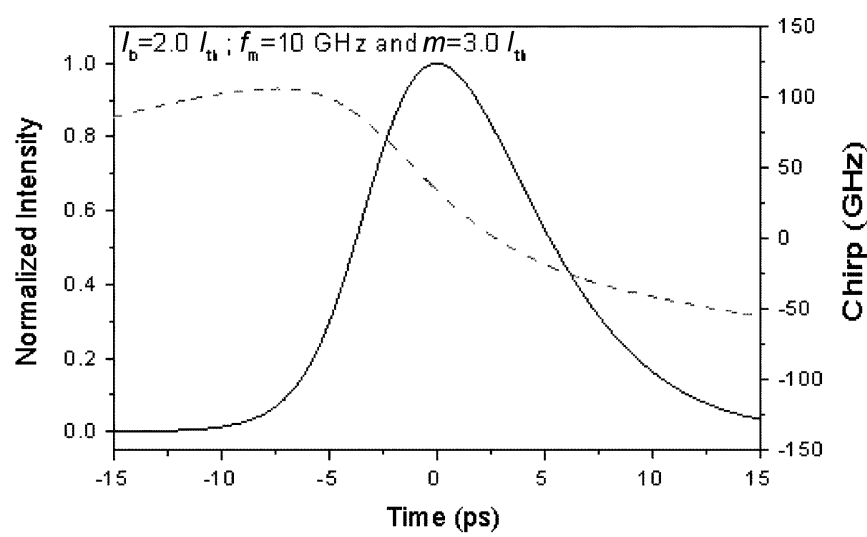

(a)

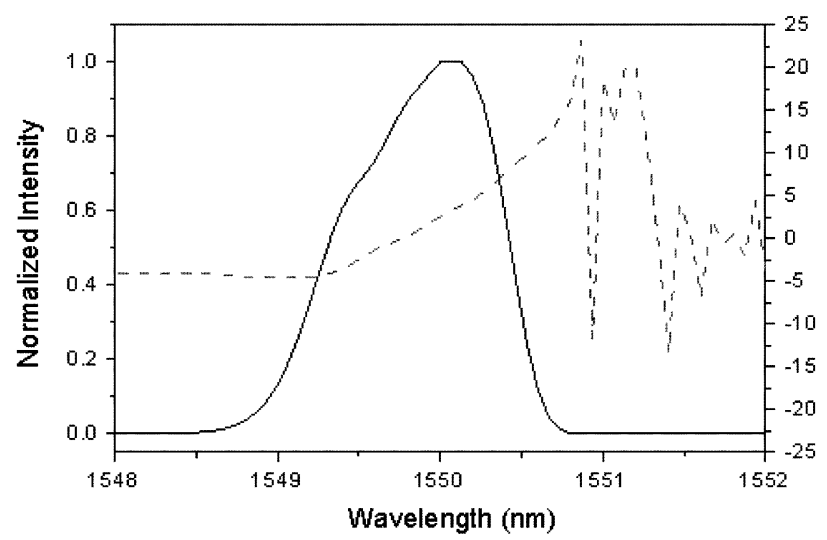

(b)

Fig. 10. Characteristics of the optical pulses from the gain-switched laser. (a) The intensity (solid line) and the chirp (dashed line) of the pulse. (b) Pulse spectrum (solid line) and its corresponding group delay (dashed line).

taken from (1) and (2) by setting the left-hand side of the equations to zero. A trajectory of at least $50 \mathrm{~ns}$ was discarded to allow for transients to die out.

Fig. 10(a) presents the intensity and the chirp of the gainswitched optical pulses when the modulation amplitude is set to $3 I_{\text {th }}$, which corresponds to experimental RF modulation level. The spectrum and group-delay profile of the gain-switched pulse is also shown in Fig. 10(b). The instantaneous frequency chirp $\delta v(t)$ (in gigahertz) across the pulse is directly obtained from the phase $\phi(t)$ by $\delta v(t)=(1 / 2 \pi)(d \varphi / d t)$. It is noted that that the 9.5-ps pulse generated exhibits a TBP of 1.3, which gives evidence of a large frequency chirp across the pulse. Moreover, as presented experimentally, the profile of the frequency chirp is similar and becomes nonlinear in the wings of the pulse. As explained previously, two types of dispersion compensation are compared in this article: one that compensates only the linear part of the pulse's group delay, whereas the other compensates the entire pulse's group delay. Figs. 11(a) and 12(a) show groupdelay and reflection profiles of the two filters used. The intensity and chirp profiles of the gain-switched pulses after reflection on the linearly and the nonlinearly chirped FBG are shown in Figs. 11(b) and 12(b), respectively.

In both cases, the reflection profile of the grating is set to have a nonlinear response to obtain a Gaussian spectral output. The

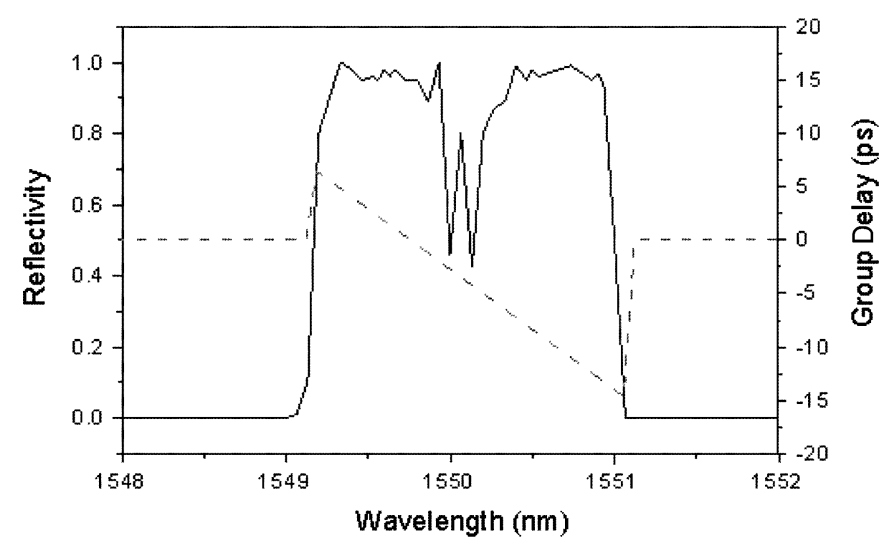

(a)

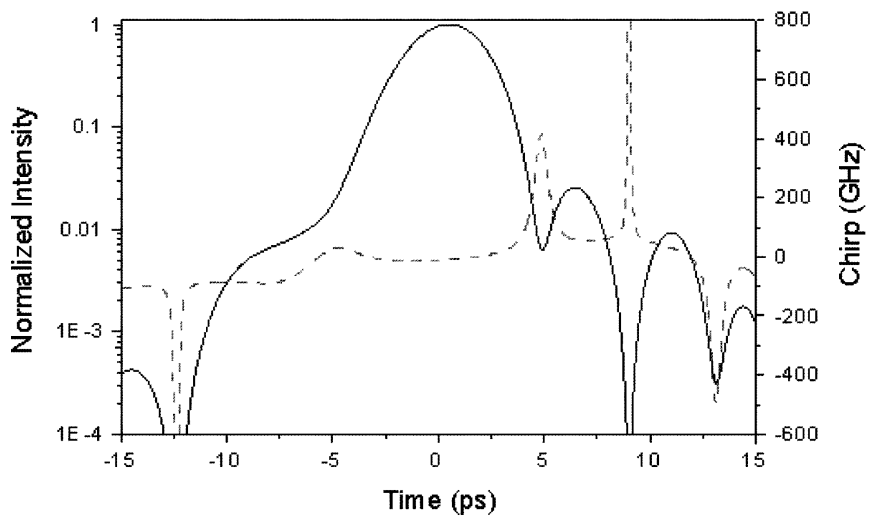

(b)

Fig. 11. (a) Reflection (solid line) and group delay (dashed line) profiles of the linearly chirped FBG with a filter response altered to achieve Gaussian output. (b) Intensity (solid line) and chirp (dashed line) of gain-switched pulses after reflection on this FBG.

use of an FBG with a group delay opposite to that of the gainswitched laser allows us to achieve transform-limited pulses. In the example used in the simulations, a 3.4-ps pulse is achieved, and there are no pedestals on the wings of the pulse. However, if the group delay of the FBG is opposite to the linear fit of the pulse's group delay, the frequency chirp across the center of the pulse is eliminated but the nonlinear chirp remains visible in the wings of the pulse. As a consequence, the 4-ps pulse is characterized by a TBP of 0.54 and contains pedestals around $16 \mathrm{~dB}$ down from the peak of the pulse as shown in Fig. 11(b). These numerical results reproduce the experimental features: compensation of only the linear portion of the chirp results in significant pedestals in the leading and trailing edges of the pulse due to the remaining nonlinear chirp.

Using our simulation model, we subsequently went on to examine the effect of using a nonlinearly chirped fibre grating with a reflection profile that is square topped and does not affect the shape of the spectrum. The grating profile and resulting output pulses are displayed in Fig. 13. As can be seen, the output pulse is characterized by a TBP of 0.57 and the pedestals are reduced around $21 \mathrm{~dB}$ down from the peak of the pulse [see Fig. 13(b)]. This result clearly shows that to obtain optimum pulses having pedestal suppression greater than $30 \mathrm{~dB}$ [28], it is 


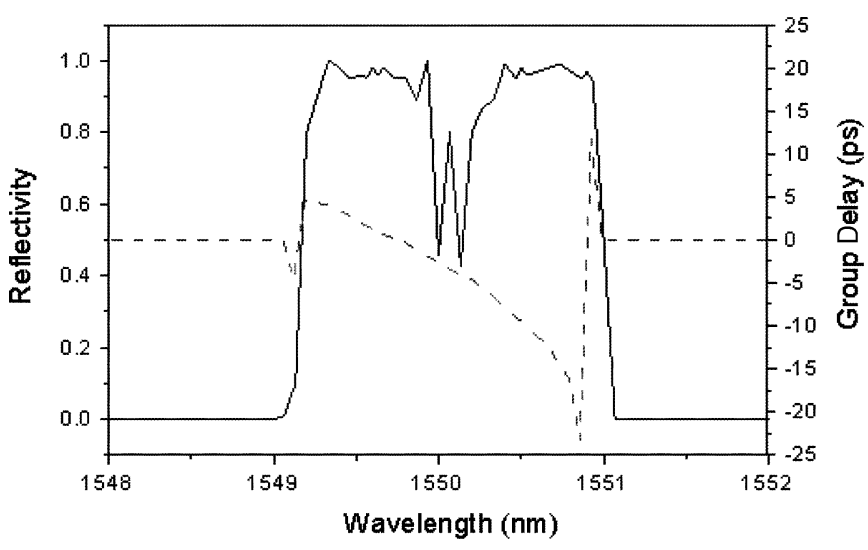

(a)

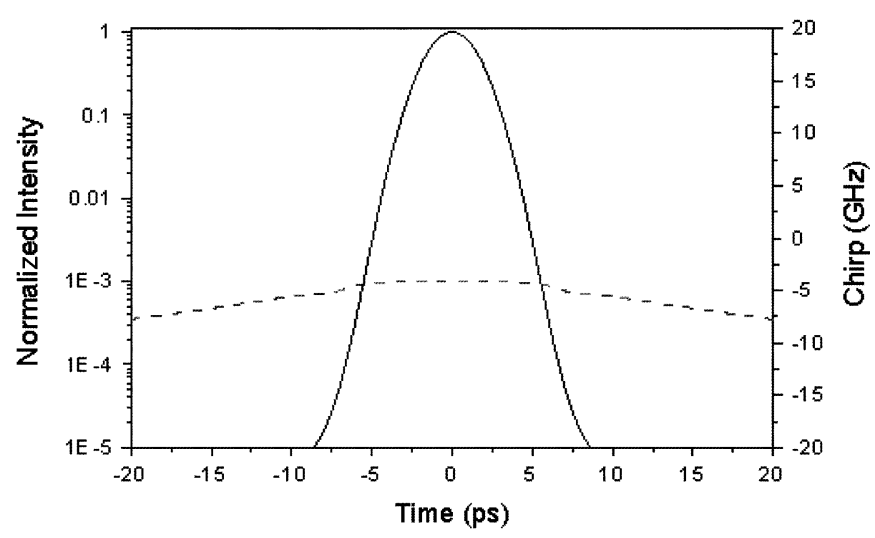

(b)

Fig. 12. (a) Reflection (solid line) and group delay (dashed line) profiles of the nonlinearly chirped fiber Bragg grating with a filter response altered to achieve Gaussian output. (b) Intensity (solid line) and chirp (dashed line) of gain-switched pulses after reflection on this FBG.

necessary to compensate for both the nonlinear chirp of the gainswitched laser and the non-Gaussian spectral output. Another possible method that we investigated through simulation, to reduce the height of the pulse pedestals obtained when a linearly chirped fibre grating is used, is to reduce the bandwidth of the FBG filter. By altering the filter bandwidth, it should be possible to filter out and eliminate the edges of the spectrum where the group delay becomes nonlinear. For this simulation, we used an FBG having a group delay that was the inverse of the linear group delay across the center of the gain-switched pulse, and a reflection profile that was set to obtain a Gaussian spectral output. We then proceeded to reduce the bandwidth of the FBG.

Fig. 14 displays the evolution of both the pedestal and the TBP of the pulse when the FBG's bandwidth is varied (as a percentage of the bandwidth of the spectrum from the gainswitched laser). When the FBG is characterized by a bandwidth that corresponds to half the pulse's bandwidth, the pedestals are reduced $28.5 \mathrm{~dB}$ down from the peak of the pulse. The resulting TBP is around 0.47 but the pulsewidth has been broadened to 7 ps. Thus, although this technique does greatly reduce the level of the pedestals, this is achieved at the expense of pulse duration, which is significantly increased.

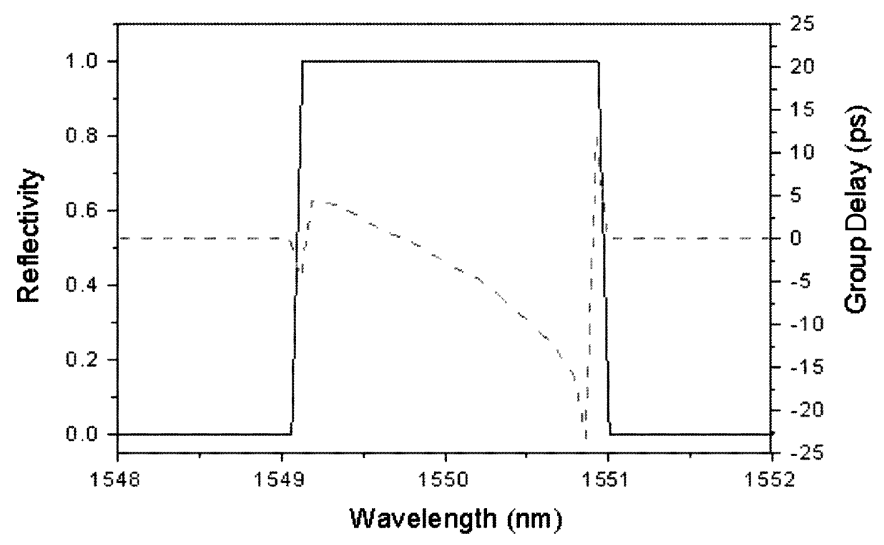

(a)

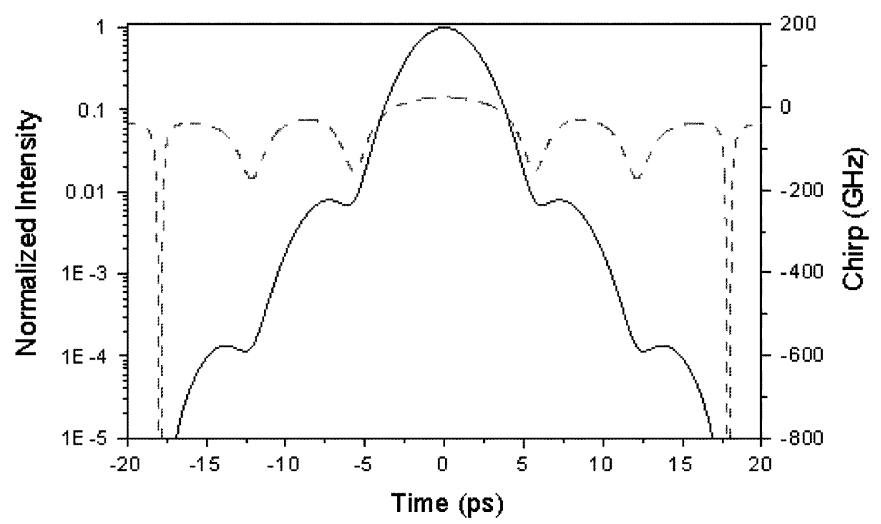

(b)

Fig. 13. (a) Reflection (solid line) and group delay (dashed line) profiles of the nonlinearly chirped fiber Bragg grating with a square filter response. (b) Intensity (solid line) and chirp (dashed line) of gain-switched pulses after reflection on this FBG.

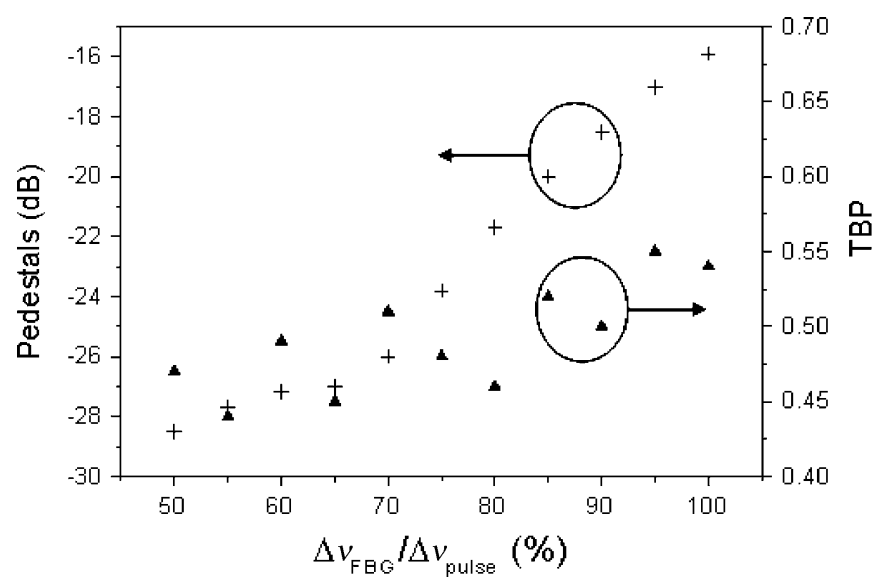

Fig. 14. Evolution of the pedestals and the time-bandwidth product of the pulses when the bandwidth of the linearly chirped fiber Bragg grating is varied.

\section{CONCLUSION}

We have demonstrated the generation of near transformlimited 3.5-ps gain-switched pulses that exhibit an excellent TPSR by using an NC FBG. The initial gain-switched pulses display a large nonlinear chirp across the wings of the pulse, and by using the measurement technique of FROG, the chirp across the pulses has been completely characterized. From this 
complete characterization, we subsequently design and fabricate a nonlinearly chirped fiber Bragg grating that has a nonlinear group-delay profile opposite to the group delay of the input pulse. The fabricated grating also has a nonlinear reflection profile to optimize the output spectrum of the generated pulses. The resultant output pulses display excellent temporal and spectral purity, which would make this pulse source ideal for use in $80 \mathrm{~Gb} / \mathrm{s}$ OTDM systems. Moreover, the experimental results were compared to a numerical analysis of the rate equations, which were found to validate the experimental results, including the reduction of the pedestals when both a specific reflective profile (to compensate for the asymmetry of the pulse spectrum) and a nonlinear group delay (to compensate for the group delay of the gain-switched laser) are used. The rate-equation analysis was utilized to investigate a possible improvement of the results obtained with a linearly chirped FBG by decreasing the FBG's bandwidth, but the reduction of the pedestals resulted in a broadening of the pulse.

\section{REFERENCES}

[1] H. Kogelnik, "High-capacity optical communications: Personal recollections,"IEEE J. Sel.Topics Quantum Electron., vol. 6, no. 6, pp. 1279-1286, Nov./Dec. 2000.

[2] W. S. Lee, V. Filsinger, L. Klapproth, H. G. Bach, and A. Beling, "Implementation of an $80 \mathrm{GBit} / \mathrm{s}$ Full ETDM multi-format ASK optical transmitter," in Proc. Eur. Conf. Optical Communications, Glasgow, U.K., Sep. 2005, vol. 3, pp. 383-384.

[3] J. P. Turkiewicz, E. Tangdiongga, G. Lehmann, H. Rohde, W. Schairer, Y. R. Zhou, E. S. R. Sikora, A. Lord, D. B. Payne, G.-D. Khoe, and H. de Waardt, "160 Gb/s OTDM networking using deployed fiber," IEEE $J$. Lightw. Technol., vol. 23, no. 1, pp. 225-235, Jan. 2005.

[4] M. Saruwatari, "All-optical signal processing for terabit/second optical transmission," IEEE J. Sel. Topics Quantum Electron., vol. 6, no. 6, pp. 1363-1374, Nov./Dec. 2000.

[5] B. Mikkelsen, C. Ramussen, P. Mamyshev, F. Liu, S. Dey, and F. Rosca, "Deployment of $40 \mathrm{~Gb} / \mathrm{s}$ systems: Technical and cost issues," in Proc. Optical Fiber Communications Conf., Los Angeles, CA, Oct. 2004, vol. 2, p. 3 .

[6] A. Belahlou, S. Bickham, D. Chowdhury, P. Diep, A. Evans, J. M. Grochocinski, P. Han, A. Kobyakov, S. Kumar, G. Luther, J. C. Mauro, M. Yihong, M. Mlejnek, M. S. K. Muktoyuk, M. T. Murtagh, S. Raghavan, V. Ricci, A. Sevian, N. Taylor, S. Tsuda, M. Vasilyev, and L. Wang, "Fibre design considerations for $40 \mathrm{~Gb} / \mathrm{s}$ systems," IEEE J. Lightw. Technol., vol. 20, no. 12, pp. 2290-2305, Dec. 2002.

[7] R. Ludwig, U. Feiste, E. Dietrich, H. G. Weber, D. Breuer, M. Martin, and F. Küppers, "Experimental comparison of $40 \mathrm{Gbit} / \mathrm{s} \mathrm{RZ}$ and NRZ transmission over standard single mode fibre," Electron. Lett., vol. 35, no. 25, pp. 2216-2218, Dec. 1999.

[8] M. I. Hayee and A. E. Willner, "NRZ versus RZ in $10-40 \mathrm{~Gb} / \mathrm{s}$ dispersionmanaged WDM transmission systems," IEEE Photon. Technol. Lett., vol. 11, no. 8, pp. 991-993, Aug. 1999.

[9] C. R. S. Fludger, Y. Zhu, V. Handerek, and R. J. Mears, "Impact of MPI and modulation format on transmission systems employing distributed Raman amplification,” Electron. Lett., vol. 37, no. 15, pp. 970-972, Jul. 2001.

[10] P. J. Winzer, M. Pfennigbauer, M. M. Strasser, and W. R. Leeb, "Optimum filter bandwidths for optically preamplified NRZ receivers," IEEE J. Lightw. Technol., vol. 19, no. 9, pp. 1263-1273, Sep. 2001.

[11] R. G. M. P. Koumans and R. Van Roijen, "Theory for passive modelocking in semiconductor laser structures including the effects of selfphase modulation, dispersion, and pulse collisions," IEEE J. Quantum Electron., vol. 32, no. 3, pp. 478-492, Mar. 1996.

[12] J. M. Roth, T. G. Ulmer, N. W. Spellmeyer, S. Constantine, and M. E. Grein, "Wavelength-tunable $40-\mathrm{GHz}$ picosecond harmonically modelocked fiber laser source," IEEE Photon. Technol. Lett., vol. 16, no. 9, pp. 2009-2011, Sep. 2004.

[13] K. Y. Lau, "Gain switching of semiconductor injection lasers," Appl. Phys. Lett., vol. 52, no. 4, pp. 257-259, Jan. 1988.
[14] L. P. Barry, R. F. O’Dowd, J. Debeau, and R. Boittin, "Tunable transform limited pulse generation using self-injection locking of an FP laser," IEEE Photon. Technol. Lett., vol. 5, no. 10, pp. 1132-1134, Oct. 1993.

[15] J. M. Dudley, L. P. Barry, J. D. Harvey, M. D. Thomson, B. C. Thomsen, P. G. Bollond, and R. Leonhardt, "Complete characterization of ultrashort pulse sources at $1550 \mathrm{~nm}$," IEEE J. Quantum Electron., vol. 35, no. 4, pp. 441-450, Apr. 1999.

[16] K. A. Ahmed, H. F. Liu, N. Onodera, P. Lee, R. S. Tucker, and Y. Ogawa, "Nearly transform limited pulse (3.6 ps) generation from gain-switched $1.55 \mu \mathrm{m}$ distributed feedback laser by using fibre compression technique," Electron. Lett., vol. 29, no. 1, pp. 54-56, Jan. 1993.

[17] B. J. Eggleton, P. A. Krug, L. Poladian, K. A. Ahmed, and H. F. Liu, "Experimental demonstration of compression of dispersed optical pulses by reflection from self-chirped optical fibre Bragg gratings," Opt. Lett., vol. 19, no. 12, pp. 877-879, Jun. 1994.

[18] P. Gunning, J. K. Lucek, D. G. Moodie, K. Smith, R. P. Davey, S. V. Chernikov, M. J. Guy, J. R. Taylor, and A. S. Siddiqui, "Gain-switched DFB laser diode pulse source using continuous wave light injection for jitter suppression and an electroabsorption modulator for pedestal suppression," Electron. Lett., vol. 32, no. 11, pp. 1010-1011, May 1996.

[19] D.-S. Seo, D. Y. Kim, and H.-F. Liu, "Timing jitter reduction of gainswitched DFB laser by external injection seeding," Electron. Lett., vol. 32, no. 1, pp. 44-45, Jan. 1996.

[20] A. Clarke, P. Anandarajah, D. Reid, G. Edvell, L. P. Barry, and J. Harvey, "Optimized pulse source for $40 \mathrm{Gbit} / \mathrm{s}$ systems based on a gain-switched laser diode in conjunction with a non-linearly chirped grating," IEEE Photon. Technol. Lett., vol. 17, no. 1, pp. 196-198, Jan. 2005.

[21] R. Trebino, K. W. DeLong, D. N. Fittinghoff, J. N. Sweetser, M. A. Krumbugel, and B. A. Richman, "Measuring ultrashort laser pulses in the time-frequency domain using frequency-resolved optical gating," Rev. Sci. Instrum., vol. 68, pp. 3277-3295, May 1997.

[22] S. Nogiwa, Y. Kawaguchi, H. Ohta, and Y. Endo, "Generation of gainswitched optical pulses with very low timing jitter by using external CWlight injection seeding," Electron. Lett., vol. 36, no. 3, pp. 235-236, Feb. 2000.

[23] A. Weiner, "Effect of group velocity mismatch on the measurement of ultrashort optical pulses via second harmonic generation," IEEE J. Quantum Electron., vol. 19, no. 8, pp. 1276-1283, Aug. 1983.

[24] A. Rosenthal and M. Horowitz, "Inverse scattering algorithm for reconstructing strongly reflecting fiber Bragg gratings," IEEE J. Quantum Electron., vol. 39, no. 8, pp. 1018-1026, Aug. 2003.

[25] J. Skaar and O. H. Waagaard, "Design and characterization of finite-length fiber gratings," IEEE J. Quantum Electron., vol. 39, no. 10, pp. 1238-1245, Oct. 2003.

[26] R. Feced, M. N. Zervas, and M. A. Muriel, "An efficient inverse scattering algorithm for the design of non-uniform fibre Bragg gratings," IEEE $J$. Quantum Electron., vol. 35, no. 8, pp. 1105-1115, Aug. 1999.

[27] C. Knothe and E. Brinkmeyer, "Reset-free phase shifter in a Sagnac-type interferometer for control of chirp and apodization of Bragg gratings," presented at the BGPP, Monterey, CA, 2003, Paper TuB3.

[28] P. L. Mason, A. Wonfor, D. D. Marcenac, D. G. Moodie, M. C. Brierley, R. V. Penty, I. H. White, and S. Bouchoule, "The effects of pedestal suppression on gain switched laser sources for $40 \mathrm{Gbit} / \mathrm{s}$ OTDM tranmission," in Proc. 10th Annu. Meeting IEEE LEOS, Nov. 10-13, 1997, vol. 1, pp. 289-290.

[29] R. A. Gabel and R. A. Roberts, Signals and Linear Systems. New York: Wiley, 1987.

[30] H. Statz and G. A. deMars, Quantum Electronics. New York: Columbia Univ. Press, 1960.

[31] G. P. Agrawal and N. K. Dutta, Long Wavelength Semiconductor Lasers. New York: Van Nostrand and Reinhold, 1986.

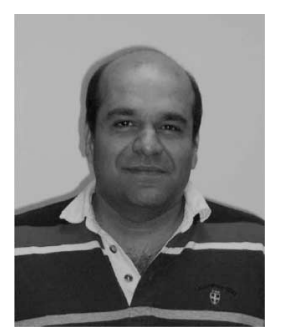

P. M. Anandarajah (S'00-M'04) received the B.Eng. degree in electronic engineering from the University of Nigeria, Nsukka, Nigeria, in 1992, and the M.Eng. and Ph.D. degrees from Dublin City University, Dublin, Ireland, in 1998 and 2003, respectively.

From 1993 to 1997, he was an Instructor/Maintenance Engineer in the Aeronautical Telecommunications Department, Nigerian College of Aviation Technology (NCAT), Zaria, Nigeria. Since September 2003, he has been a Postdoctoral Researcher with the Radio and Optical Communications Laboratory at the Research Institute for Networks and Communications Engineering (RINCE), Dublin City University. 


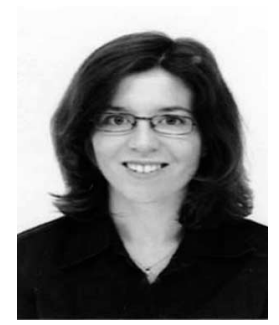

C. Guignard received the Eng. degree in optronics in 2001, the Masters degree in Sciences and Techniques of Communications (D.E.A.) in optics communications, also in 2001, and the Ph.D. degree in optronics in 2005 from the University of Rennes, Rennes, France.

Since April 2005, she has been a Postdoctoral Researcher in the Radio and Optical Communications Laboratory, Research Institute for Networks and Communications Engineering (RINCE), Dublin City University, Dublin, Ireland. Her research work includes optical pulse generation using actively mode-locked semiconductor laser diode submitted to nonlinear and filtered feedback and the effect of optical injection on these pulses.

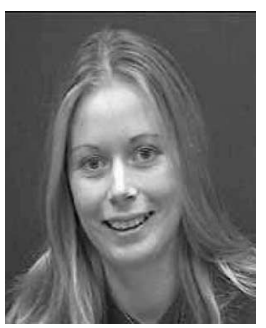

A. Clarke (S'03) received the B.Eng. degree in electronic engineering in 2002 from Dublin City University, Dublin, Ireland, where she is currently pursuing the Ph.D. degree in optical communications. The main topic of her research is high-speed alloptical processing using semiconductor optical amplifiers (SOAs).

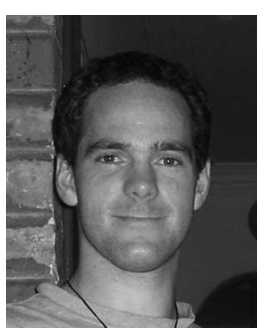

D. Reid received the B.Tech. degree in optoelectronics and the M.Sc. degree in physics in 2000 and 2002, respectively, from the University of Auckland, Aukland, New Zealand, where he is currently working toward the Ph.D. degree in physics (specializing in optical communications).

His research interests include the development of techniques for the characterization of ultrashort optical pulses, in particular, the use of FROG and other linear spectrographic techniques for real-time measurement of optical communication pulses. Other areas of interest include the design of optimized pulse sources and compressors, the latter being made possible by accurate pulse characterization.

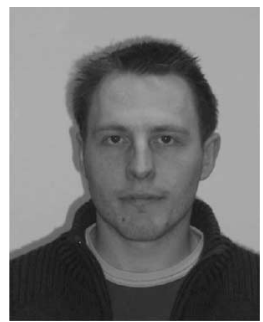

M. Rensing received the B.Sc. (honors) degree in physics from the University of Hull, Hull, U.K., in 2001, and the H.Dip. degree in applied physics from University College Cork (UCC), Cork, Ireland, in 2004. He is currently working toward the M.Eng. degree in optical communications at Dublin City University, Dublin, Ireland.

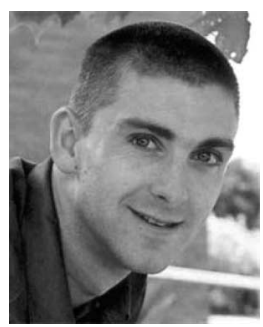

L. P. Barry (M'98) received the B.E. degree in electronic engineering and the M.Eng.Sc. degree in optical communications from University College Dublin, Dublin, Ireland, in 1991 and 1993, respectively, and the Ph.D. degree from the University of Rennes, Rennes, France.

From February 1993 to January 1996, he was a Research Engineer in the Optical Systems Department of France Telecom's Research Laboratories (CNET), Lannion, France. In February 1996, he joined the Applied Optics Centre, University of Auckland, Auckland, New Zealand, as a Research Fellow. In March 1998, he took up a lecturing position in the School of Electronic Engineering, Dublin City University, Dublin, Ireland, where he has since developed the Radio and Optical Communications Laboratory.

G. Edvell is a Senior Research and Development Engineer with Redfern Optical Components, Eveleigh, Australia.

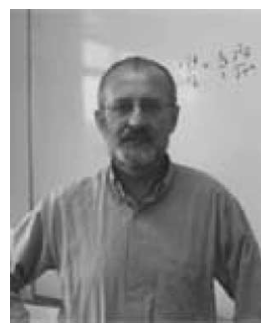

J. D. Harvey (M'76) received the B.Sc. and M.Sc. degrees from the University of Auckland, Auckland, New Zealand, in 1965 and 1967, respectively, and the $\mathrm{Ph} . \mathrm{D}$. degree from the University of Surrey, Surrey, U.K.

In 1970, he joined the University of Auckland, where he now holds a Chair in the Physics Department. His research interests include nonlinear fiber optics, ultrafast processes, and mode-locked lasers.

Prof. Harvey is a Fellow of the New Zealand Institute of Physics and a member of the Optical Society of America and the Australian Optical Society. 